\title{
Color Image Compression using hybrid HAAR-DCT wavelet in Different color spaces
}

\author{
H. B. Kekre ${ }^{1}$, Tanuja Sarode ${ }^{2}$, Prachi Natu ${ }^{3}$ \\ ${ }^{1,3}$ MPSTME, NMIMS University, Mumbai, India; \\ ${ }^{2}$ TSEC, Mumbai University, India; \\ hbkekre@yahoo.com,tanuja_0123@yahoo.com,prachi.natu@yahoo.com
}

\begin{abstract}
This paper compares digital image compression in various color spaces using Hybrid Haar wavelet transform. As DCT has high energy compaction property, it is combined with Haar transform generating Haar-DCT hybrid wavelet transform. Different component sizes are used and error at different compression ratios up to 32 is observed. At higher compression ratios 1616 component pair gives minimum error. This pair is further used to compress images in different color spaces like RGB, KLUV, YUV, YIQ, XYZ and YCbCr. KLUV color space show considerably less Root Mean Square Error (RMSE) and Mean Absolute Error (MAE) than other color spaces. Average Fractional Change in Pixel Value (AFCPV) in KLUV color space is nearly half than other color spaces. RMSE, MAE these measures are not sufficient as it give perceived error to human visual system. Hence other error metric Structural Similarity Index (SSIM) is used to observe performance superiority of various color spaces. SSIM in KLUV and RGB color space is equal having value 0.994 which is closest to one indicating better but similar image quality in both color spaces. This observation cannot be emphasized using traditional error metrics like RMSE, MAE and AFCPV.
\end{abstract}

Keywords: Image Compression, Hybrid Wavelet Transform, SSIM, AFCPV

\section{Introduction}

Digital images are integral part of multimedia data and used extensively in many applications today. Also large amount of image data is transferred over internet. To facilitate easy storage and reduction in bandwidth required to transmit this data, compression of data becomes necessary. Compression reduces the number of bits required to represent an image. Data is compressed before storage and transmission and the receiving end it is decompressed to get original data back. If decompressed data does not show any loss of contents then it is called as lossless compression. In contrast to this if decompressed data is different than original data it means that some loss of information is there and such type of compression is called as lossy 
compression. Lossy compression gives higher compression ratio than lossless compression. In image compression loss of redundant information not visible to human visual system is acceptable. Hence lossy compression is preferred for images.

Many transform based image compression methods are available in literature. Still in last two decades use of wavelets is emphasized for image compression. Wavelet transform gives time and frequency analysis of a signal. It is good in time resolution of high frequencies, while for slowly varying functions, it is good in frequency resolution. Basic idea behind wavelet is to select function prototype called mother wavelet. Then scaled and shifted version of mother wavelet can be used to analyze the image data. Due to high energy compaction property, it gives better compression ratio than Fourier Transform, Short Term Fourier Transform (STFT) [1] and popularly used Discrete Cosine Transform (DCT) [2]. Multi-resolution analysis [3] is another important property of wavelet transform that helps to view the signal at different levels. Aim of this paper is to use hybrid wavelet transform for color image compression in different color spaces.

\section{Review of Literature}

Image compression using wavelet transform is focused by many researchers since last two decades. M. S. Abdullah and N. Subba Rao [4] have proposed comparison of different wavelets for image compression. Daubechies wavelet [5], Haar wavelet, Coiflet, Symlet and bi orthogonal Wavelet transforms have been used to compare their performances. Wei Pin Lin et al. proposed a method [6] to predict the data of high frequency from a decimated image with specified down sampling rule. It exploits the correlation between low pass and high pass outputs which can be used to compress the image and get good quality reconstructed image. Tree based wavelet image coder is proposed by Jose Oliver, Manuel P. Malumbres [7]. In this method wavelet tree is formed by logically arranging transform coefficients so as to remove the redundancy among the sub bands. Arian Maleki et al. have proposed image compression using directional wavelet transforms [8]. To avoid the drawback of quad tree partitioning, they have used new scheme of partitioning called mega quad partitioning. Shih-Chung B. Lo, Huai Li, and Matthew T. Freedman have proposed neural network based wavelet transform for image compression [9]. They have used linear convolution neural network to seek the wavelet that minimizes the error and maximizes the efficiency. Daubechies wavelet and Haar wavelet have been used by them for compression. A hybrid image compression technique implementing a four-dimensional transform combining the discrete wavelet transform and the discrete cosine transform has been proposed by E. Elharar et al. [10]. It is used for integral imaging and outperforms JPEG compression scheme applied to integral images. Image compression using dual tree complex wavelet transform is presented by Li Hui Fang et al. [11]. It uses SPIHT algorithms, which is based on not only the relationship between the bit-planes and the target bit-rate but also the relationship between the initial threshold and the target bit-rate. Combination of fractal image 
encoding and wavelet decomposition is proposed in by Xiao-Yan Xu et al. [12]. In their proposed process of encoding, the image is dealt by three level wavelet decomposition. High frequency section of the decomposed image adopts scathless predicting encoding, while the low frequency section adopts fractal compression encoding. In the process of decoding, the high frequency section carries out scathe less predicting decoding, while the low frequency section is decoded to iterative function system code which is used for subimage reconstruction. Compression using hybrid transform is presented in [13]. Kronecker product of Kekre transform with other orthogonal transforms is used and error at different compression ratios is observed. It has been observed that when DCT is combined with Kekre transform gives minimum error as DCT has high energy compaction property than other orthogonal transforms.

Simple wavelet generation method proposed by Kekre et al. [14] is used to compare performance of wavelet transforms to respective orthogonal transforms [15]. It has been observed that wavelet transforms of orthogonal transforms give better compression than respective orthogonal transform. Next advancement to wavelet transforms is hybrid wavelet transform that uses two different orthogonal transforms to generate new transformation matrix. In recent years hybrid wavelet transforms have been studied and proven to be better than wavelet transform in many image processing applications including image compression. Compression using Real Fourier-DCT hybrid wavelet transform is proposed by Kekre et al. [16]. It shows that when RealDFT and DCT are combined to generate hybrid wavelet, it gives better performance than compression obtained by individual DCT or Real DFT. It is also better than DCT wavelet transform [8] .Hybrid wavelet of Haar and non sinusoidal transforms has been proposed in [17]. Different sizes of component transforms have been tried and one giving better image quality has been selected. On the other hand Haar transform paired with sinusoidal transforms is used for image compression [18]. Haar transform paired with DCT gives superior performance than its combination with DST, Hartley and Real-DFT.

This paper is extension of our previous work based on hybrid Haar wavelet transform. Here Haar-DCT hybrid wavelet is used for compressing images in various color spaces. Different error metrics are used as RMSE is not a substantial measure to observe the performance in different color spaces.

\section{Proposed Method}

Let $\mathrm{A}$ denotes $\mathrm{MxM}$ base component transform and B denotes NxN local component transform. Then using $A$ and $B$ hybrid wavelet $T$ of $M N x M N$ size is generated using Kekre's algorithm. First ' $M$ ' rows of resultant matrix are calculated by repeating each column of ' $A$ ', ' $N$ ' times and multiplying it with each element of first row of $B$. These ' $M$ ' rows represent global characteristics in hybrid wavelet transform. Remaining rows are obtained by translating the rows of matrix $B$ and padding zeroes to it from second row onwards. These rows contribute local features of an image. Generated hybrid wavelet transform is shown below in Figure 1. 


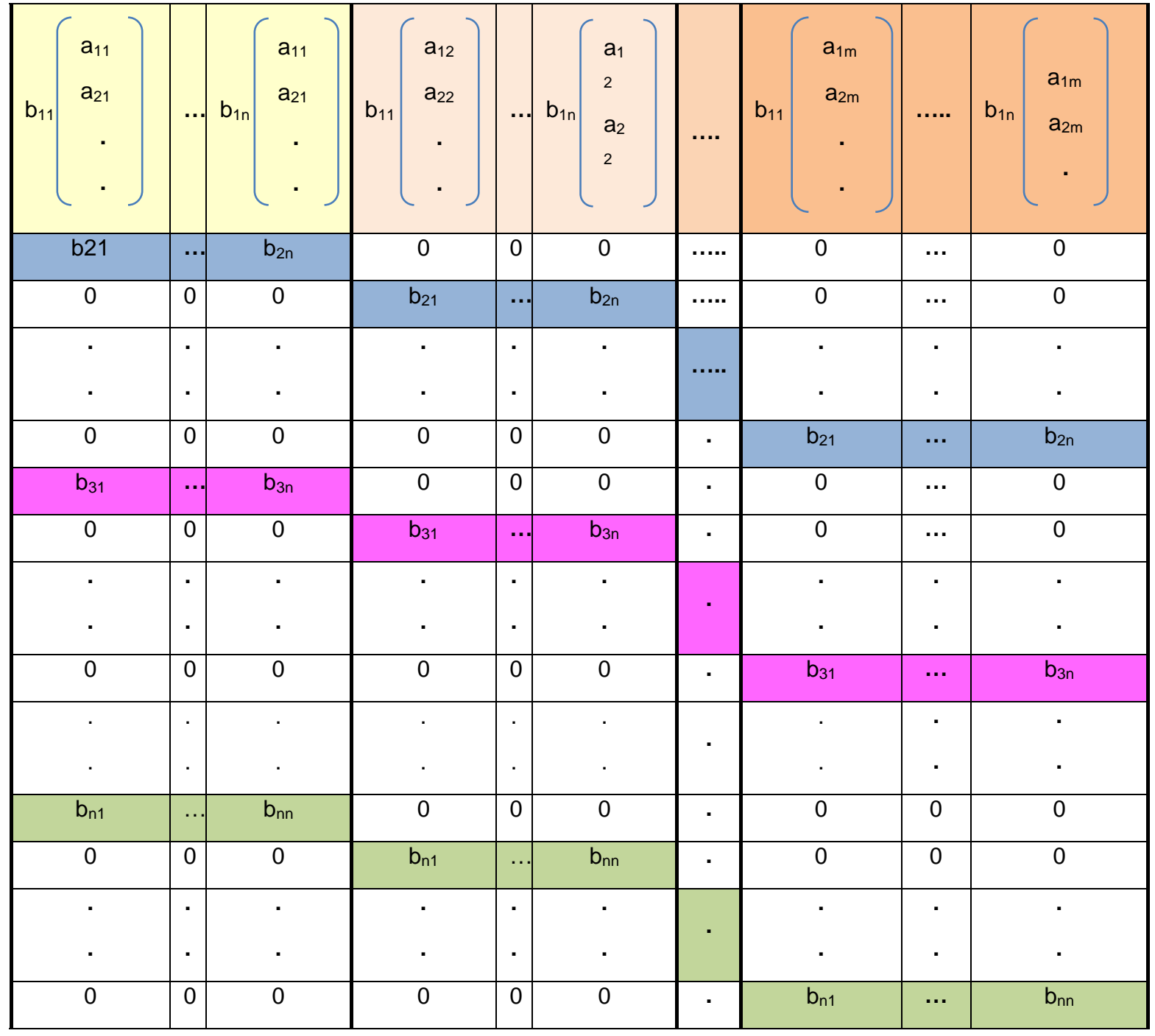

Figure 1: Generation of Hybrid Wavelet Matrix using Component Matrices A and B

Hybrid wavelet using different component size is generated and applied on images. To generate $256 \times 256$ size transformation matrix, different component sizes used are 8-32, 16-16, 32-8 and 64-4. Here 8-32 means $8 \times 8$ base transform and $32 \times 32$ local transform. Here Haar transform is used as base transform and DCT is used as local component transform to generate Haar-DCT hybrid wavelet of size $256 \times 256$.

\section{Results and Discussions}

Proposed method is applied on a set of color images belonging to different classes. Each image is of size 256x256. Execution is done on AMD dual core processor using Matlab 2012. Set of test images is shown in Figure 2. 

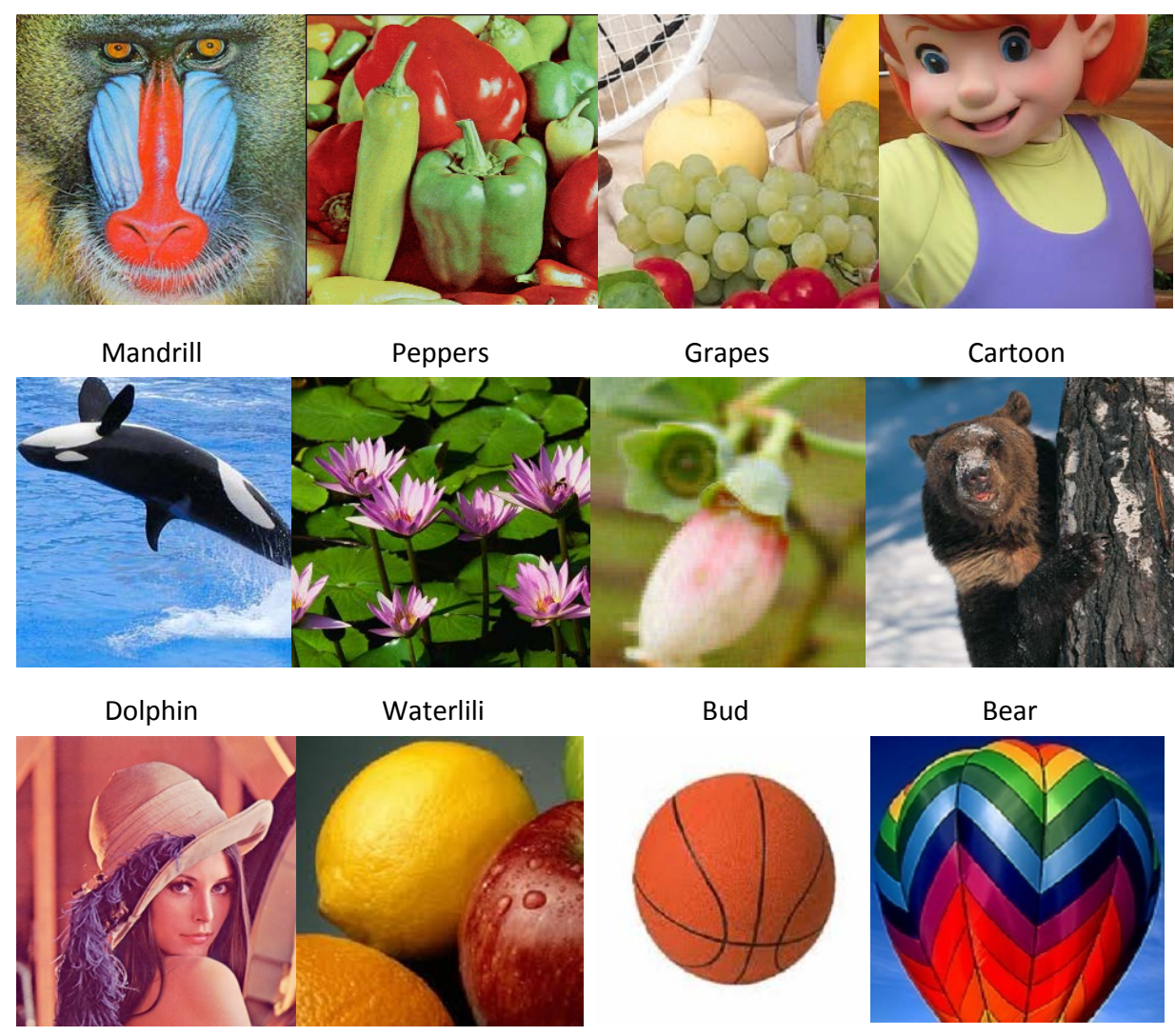

Bud

Bear
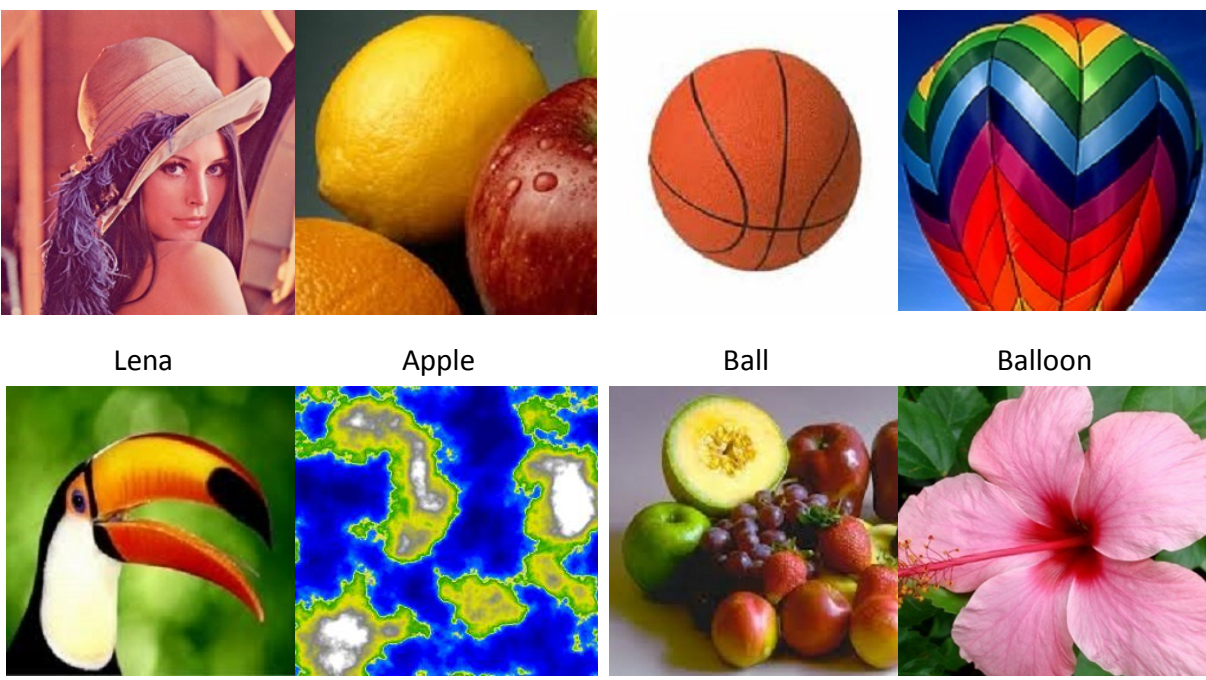

Bird

Colormap

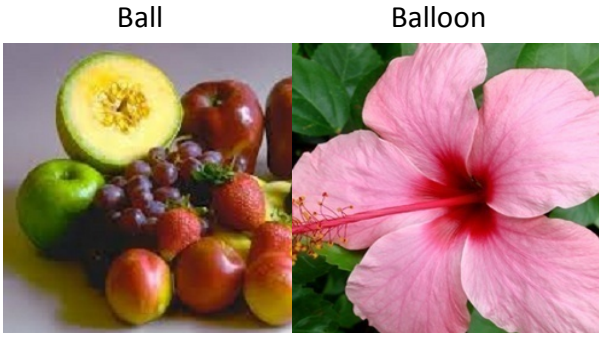

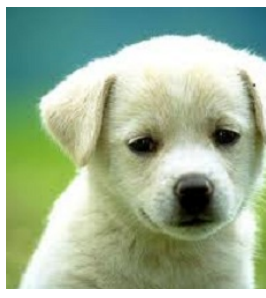

Puppy

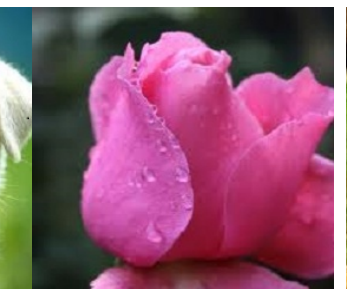

Rose
Fruits

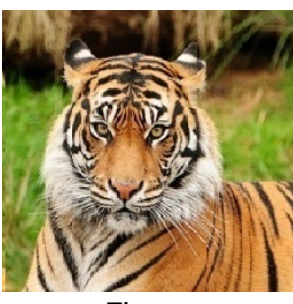

Tiger
Hibiscus

Figure 2: Test Set of Images from Different Classes

Figure 3 shows graph of RMSE versus compression ratio for Haar-DCT hybrid wavelet transform. To obtain transformation matrix of size $256 \times 256$, different component sizes like $8 \times 8$ Haar and $32 \times 32$ DCT can be selected. It is denoted as 8-32 for reading simplicity. Similarly 16-16, 32-8 and 64-4 pairs can also be used. 


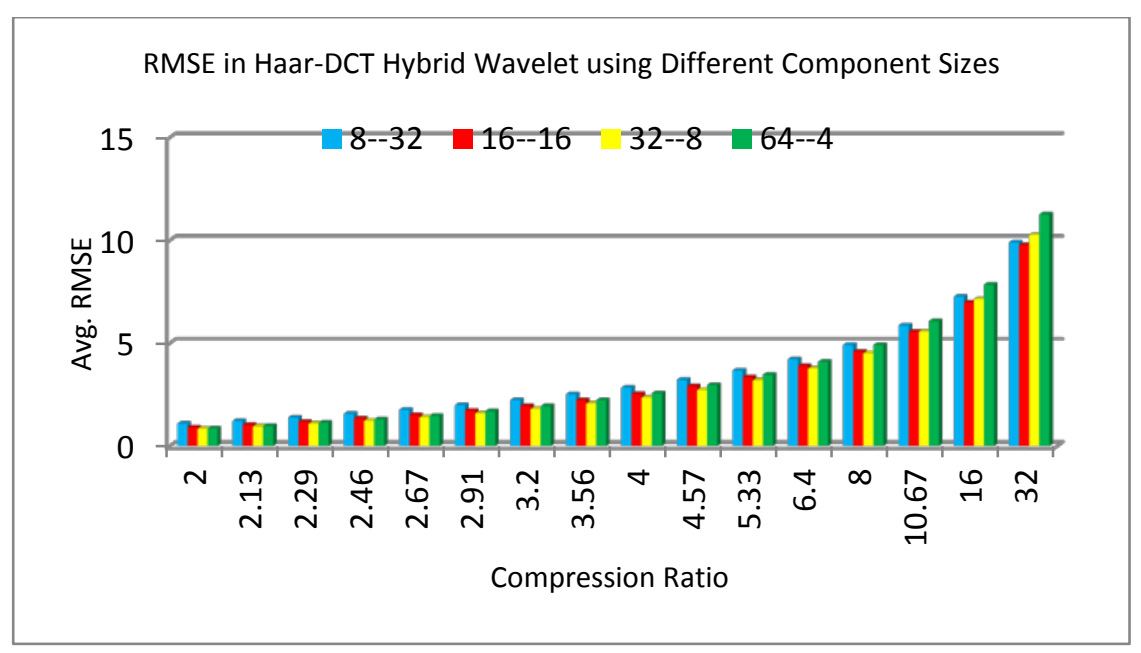

Figure 3: RMSE in Haar-DCT Hybrid Wavelet Transform With Variation in Component Sizes

It has been observed that for lower compression ratios up to 8, a pair that gives less RMSE is 328. At compression ratio 10.67 , both pairs of $16-16$ and $32-8$ give equal error and at higher compression ratios 16-16 component size give less error.

This combination is further used to compress image in different color spaces. Six different color spaces namely RGB, KLUV, XYZ, YUV, YIQ, YCbCr have been used. RMSE against compression ratio in these different color spaces is plotted in fig. 4.

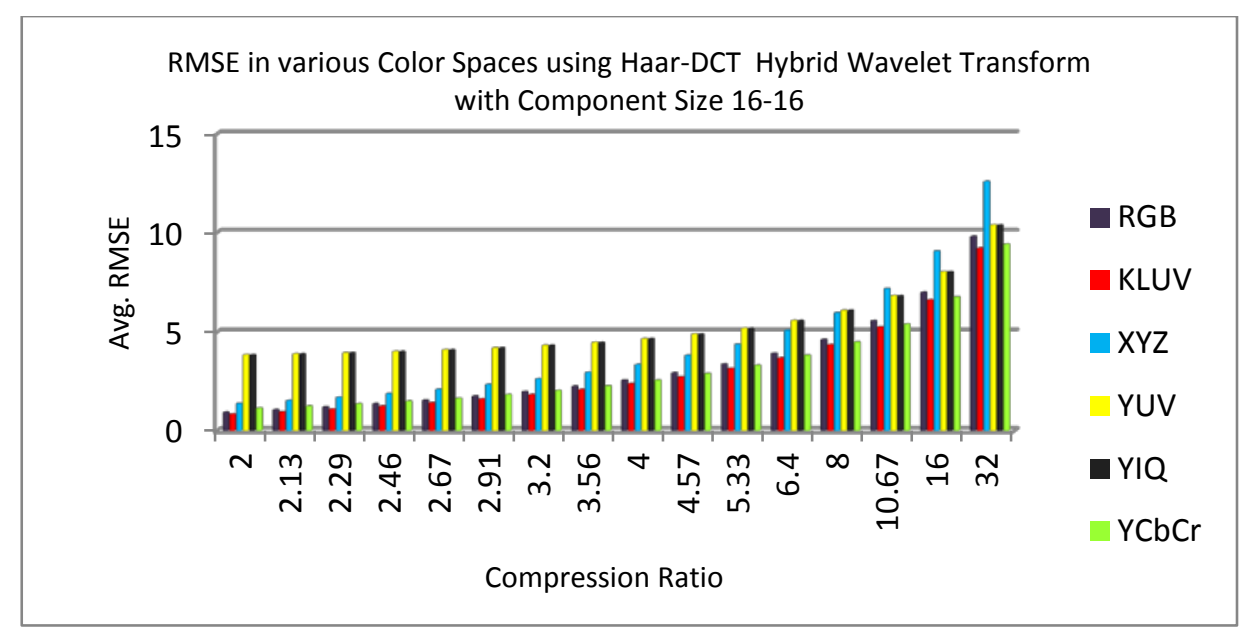

Figure 4: RMSE in various Color Spaces using Haar-DCT Hybrid Wavelet Transform with Component Size 16-

16

From Figure 4 it has been observed that, RMSE obtained in KLUV color space is less than RMSE in other color spaces for all compression ratios. Error in $\mathrm{YCbCr}$ color space is slightly more than in KLUV color space and it is followed by error in RGB color space. XYZ color space shows maximum error. YIQ and YUV color space show same error.

Further mean absolute difference between original image and reconstructed image is computed. In Figure 5, MAE at various compression ratios is plotted for different color spaces. 
Error pattern obtained is similar to that of RMSE. Lowest MAE is obtained in KLUV color space followed by MAE in YCbCr color space and then RGB color space.

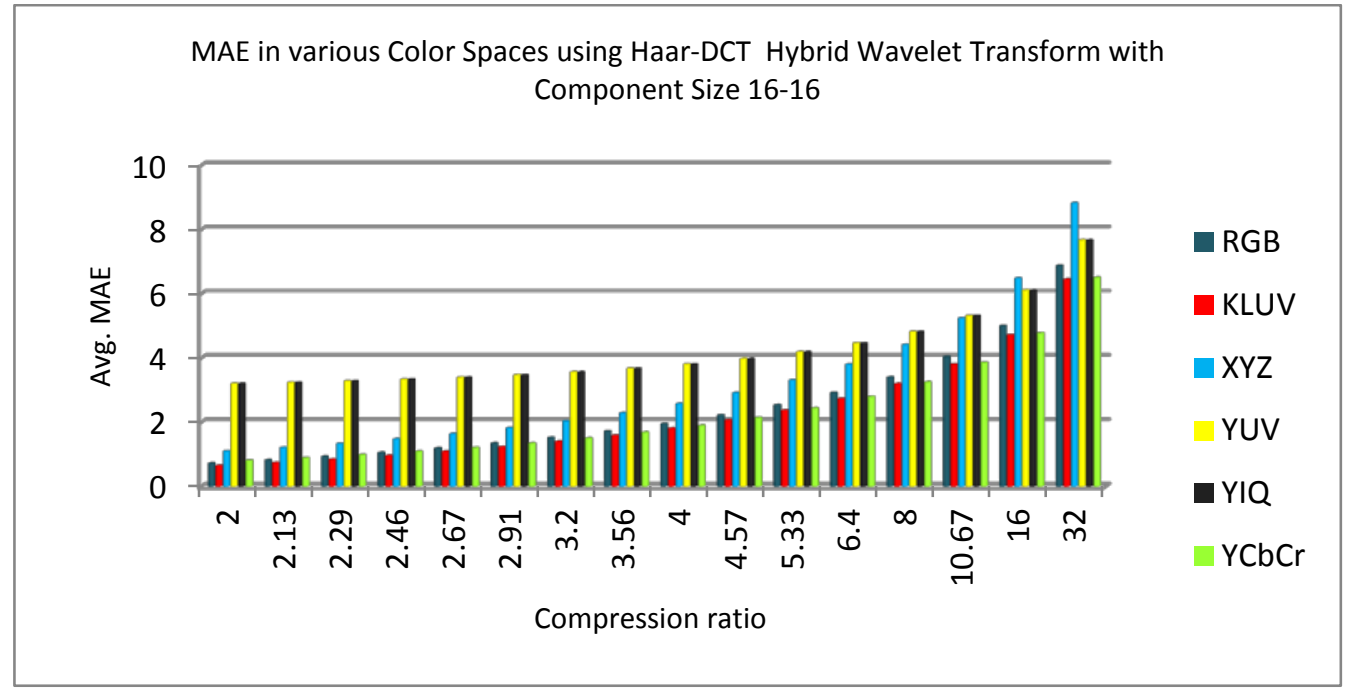

Figure 5: MAE in various Color Spaces using Haar-DCT Hybrid Wavelet Transform with Component Size 16-16 AFCPV for various color spaces is plotted in Figure 6. In KLUV color space AFCPV is nearly half of AFCPV obtained in RGB, XYZ, YUV and YIQ color spaces.

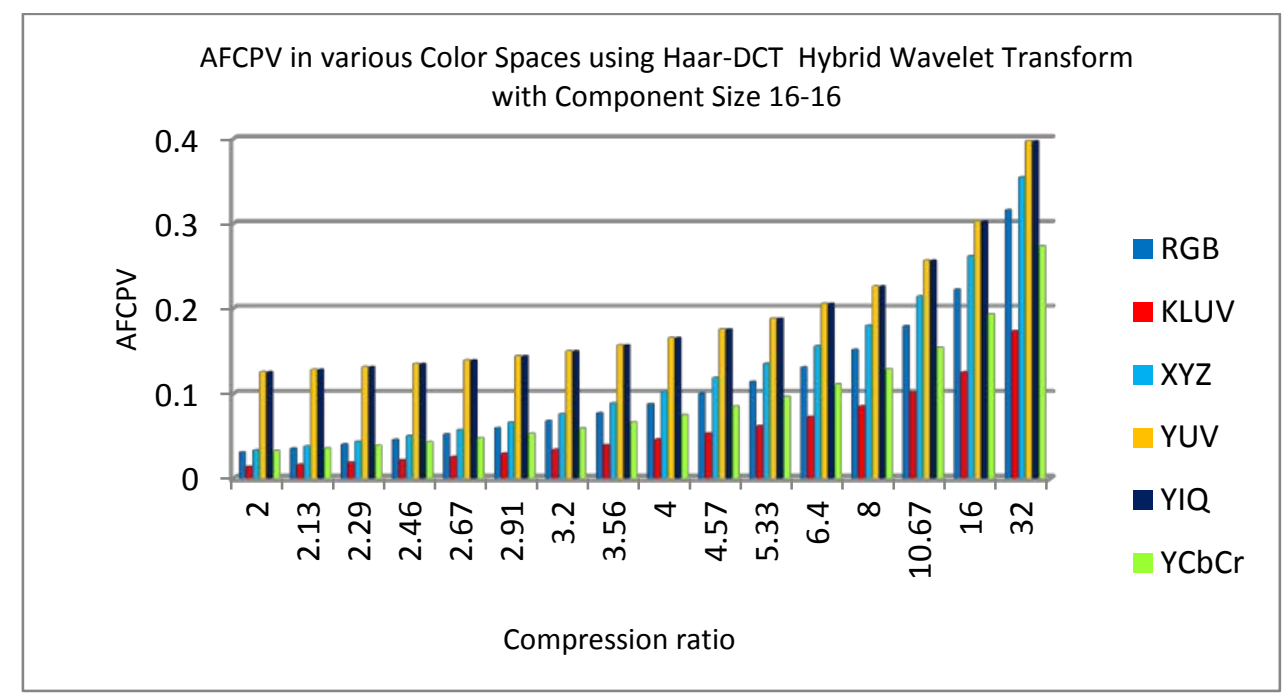

Figure 6: AFCPV in various Color Spaces using Haar-DCT Hybrid Wavelet Transform with Component Size 1616

Figure 7 shows SSIM plotted against compression ratio. SSIM considers image degradation as perceived change in structural information. Hence provides good approximation to perceived image quality. Value of SSIM ranges between -1 to 1 . It is 1 for exactly similar images. 


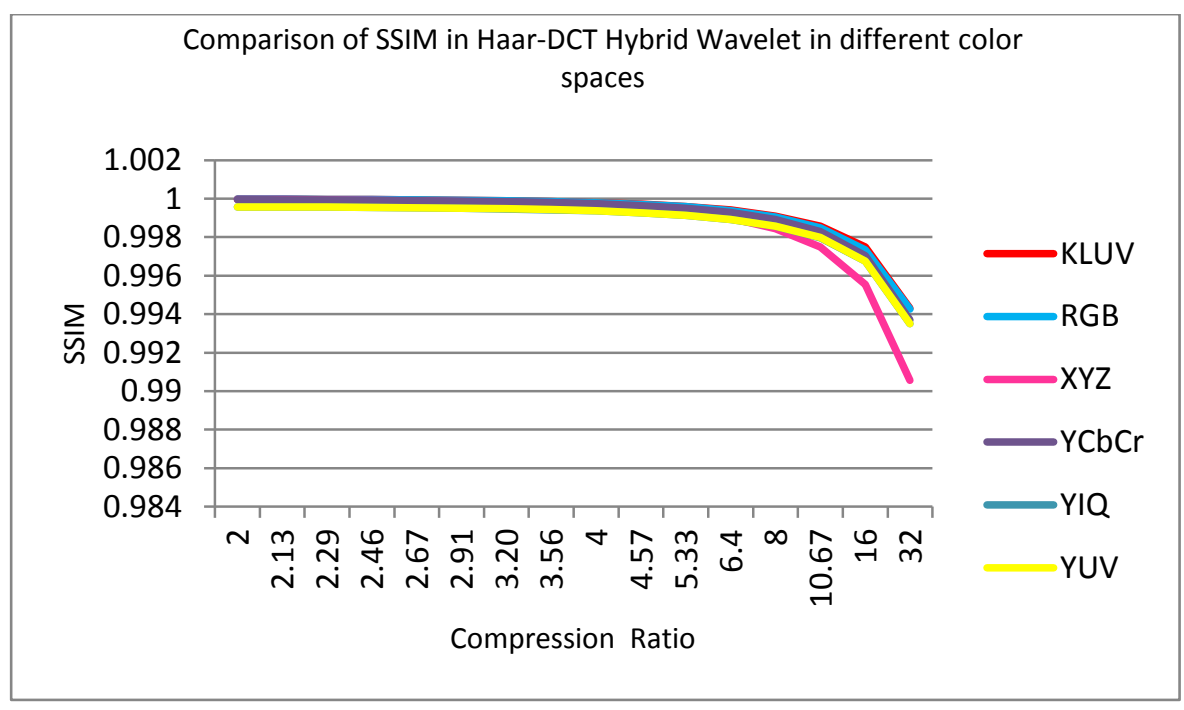

Figure 7: Comparison of SSIM in Haar-DCT Hybrid Wavelet in different color spaces

As shown in Figure 7, SSIM in KLUV color space at compression ratio 32 is 0.9943 . It indicates that reconstructed image quality is closer to original image. In RGB color space SSIM value is equal to 0.994 showing similar image quality. SSIM measure in YUV, YIQ and YCbCr color space exhibit similar performance showing value 0.993. XYZ color space shows lowest SSIM means that lower image quality.

Figure 8 shows reconstructed image 'Lena' obtained using Haar-DCT hybrid wavelet transform at compression ratios 4, 8, 16 and 32 respectively. Different color spaces used are $\mathrm{RGB}, \mathrm{YCbCr}$, $X Y Z, K L U V, Y I Q$ and YUV. SSIM value of each image is indicated below the image. From figure it is clear that, SSIM value closest to 1 indicates that reconstructed image with better quality is obtained. With increase in compression ratio SSIM value decreases. Though similar AFCPV values are obtained at specific compression ratio, difference in reconstructed image quality is highlighted by SSIM values of image at that compression ratio.

\begin{tabular}{|c|c|c|c|c|}
\hline & \multicolumn{5}{|c|}{ Compression Ratio } \\
\hline Color space & 4 & 8 & 2 & 16 \\
\hline
\end{tabular}




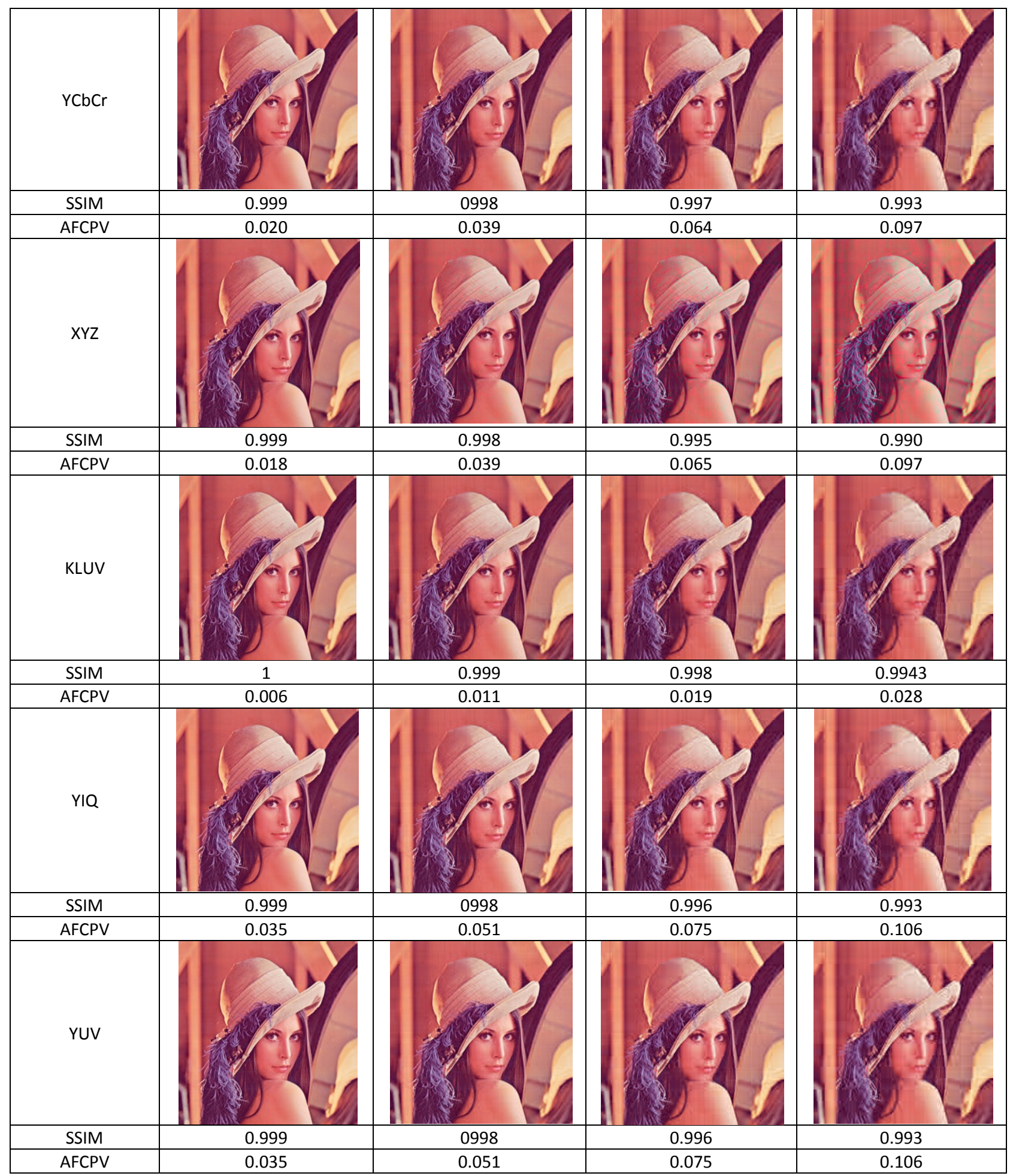

Figure 8: Reconstructed 'Lena' Image in Various Color Spaces using Haar-DCT hybrid Wavelet Transform at Different Compression Ratios

\section{Conclusion}

In this paper performance of Haar-DCT hybrid wavelet in various color spaces is compared. From different component size combinations, 16-16 component size is observed to better in 
RGB color space and is used in different color spaces. Different error metrics are used to compare the performance as RMSE gives the information about the perceived error. KLUV color space shows minimum error than other color spaces in terms of RMSE, MAE and AFCPV. Compression in KLUV color space can be better measured in terms of SSIM than RMSE, MAE and AFCPV in any color space. SSIM of 0.9943 is obtained in KLUV color space at compression ratio 32. As it is closest to 1 it indicates that reconstructed image is much similar to original image. It is closely followed by RGB color space showing slight less SSIM than KLUV color space. Though RMSE, MAE and AFCPV in KLUV color space is considerably less than respective values in RGB color space, SSIM values show negligible difference in these two color spaces. It indicates slight difference in quality of reconstructed images

\section{REFERENCES}

[1]. $\quad$ Strang G. "Wavelet Transforms Versus Fourier Transforms." Bull. Amer. Math. Soc. 28, 1993, 288-305.

[2]. N. Ahmed, T. Natarajan and K. R. Rao, "Discrete Cosine Transform", IEEE Transaction Computers, January 1974, C-23, pp. 90-93.

[3]. S. Mallat, "A Theory of Multiresolution Signal Decomposition: The Wavelet Representation," IEEE Trans. Pattern Analysis and Machine Intelligence, vol. 11, pp. 674-693, 1989.

[4]. M.S. Abdullah, N. Subba Rao, "Image Compression using Classical and Lifting based Wavelets", International Journal of Advanced Research in Computer and Communication Engineering, August 2013, Vol. 2, Issue 8, pp. 3193-3198.

[5]. Daubechies, I. "The wavelet transform, time-frequency localization and Signal analysis", IEEE Transformation and Information Theory 36: 1990, 961-1005.

[6]. Wei-Pin Lin, Chih-Ming Chen, and Yung-Chang Chen, "Image Compression with Interpolation in Wavelet Transform Domain" 2005. IEEE International Symposium on Circuits and Systems (ISCAS), 2005, Vol. 3, pp. $2084-2087$.

[7]. Jose Oliver, Manuel P. Malumbres, "Low Complexity Multi-Resolution Image Compression using Wavelet Lower Trees", IEEE Trans. on Circuits and Systems for Video Technology, Nov. 2006, Vol. 16, Issue 11, pp. 1437-1444.

[8]. Maleki A, Rajaei B, Pourreza H.R., "Rate Distortion Analysis of Directional Wavelets", IEEE Trans. on Image Processing, Feb 2012, Vol. 21 Issue 2, pp. 588-600.

[9]. Shih-Chung B. Lo, Huai Li, and Matthew T. Freedman, "Optimization of Wavelet Decomposition for Image Compression and Feature Preservation", IEEE Trans. on Medical Imaging, Vol. 22, Issue 9, Sept 2013, pp. 1141-1151. 
[10]. E. Elharar, Adrian Stern, Ofer Hadar, and Bahram Javidi, "A Hybrid Compression Method for Integral Images using Discrete Wavelet Transform and Discrete Cosine Transform", Journal Of Display Technology, September 2007, Vol. 3, No. 3, pp. 321-325.

[11]. Jingyu Yang, Wenli Xu, Qionghai Dai, Yao Wang, “Image Compression using 2D Dual Tree Discrete Wavelet Transform (DDWT)", IEEE International Symposium on Circuits and Systems, May 2007, pp. 297-300.

[12]. Xiao-Yan Xu, Chen, P., Juan Dai, “Hybrid Encoding Analysis of Fractal Image Compression Method Based on Wavelet Transform", In Proc of International Conference of Machine Learning and Cybernetics, 2008, Vol. 5, pp. 2886-2889.

[13]. H.B. Kekre, Tanuja Sarode, Prachi Natu, “Digital Image Compression using Hybrid Transform with Kekre Transform and Other Orthogonal Transforms", Journal of Computer Engineering, Jan. 2014, Vol. 16, Issue 1, Ver. III, PP 38-46.

[14]. H. B. Kekre, Tanuja Sarode, Sudeep Thepade, Sonal Shroff, "Instigation of Orthogonal Wavelet Transforms using Walsh, Cosine, Hartley, Kekre Transforms and their use in Image Compression", International Journal of Computer Science and Information Security, 2011, Vol. 9, No. 6, pp. 125-133.

[15]. H.B. Kekre, Tanuja Sarode, Prachi Natu, “ Image Compression Using Column, Row and Full Wavelet Transforms Of Walsh, Cosine, Haar, Kekre, Slant and Sine and Their Comparison with Corresponding Orthogonal Transforms". International Journal of Engineering Research and Development (IJERD), Mar.2013, Vol. 6. Issue 4, pp. 102-113.

[16]. H.B. Kekre, Tanuja Sarode, Prachi Natu, “Image Compression using Real Fourier Transform, It's Wavelet Transform and Hybrid Wavelet with DCT", International Journal of Advanced Computer Science and Applications (IJACSA), 2013, Vol. 4, No.5, pp. 41-47.

[17]. H.B. Kekre, Tanuja Sarode, Prachi Natu, "Color Image Compression using Hybrid Wavelet Transform with Haar as Base Transform", International Journal of Scientific and Research Publications, June 2014, Volume 4, Issue 6, pp. 1-13.

[18]. H.B. Kekre, Tanuja Sarode, Prachi Natu, "Performance Comparison of Hybrid Haar Wavelet Transform with Various Local Transforms in Image Compression using Different Error Metrics", International Journal of Image Processing (IJIP), July 2014, Vol.8, Issue 4, pp. 186-203 